\title{
Diffraction Mapping with a Pixelated Detector to Quantify Crystal Orientation in 3D Structures Made from 2D Materials
}

\author{
Michael C. Cao ${ }^{1}$, Joonki Suh ${ }^{2,3}$, Zhen Chen ${ }^{1}$, Elliot Padgett ${ }^{1}$, Chibeom Park ${ }^{4}$, Jiwoong Park ${ }^{2,4,5}$, \\ and David A. Muller ${ }^{1,6}$ \\ 1. School of Applied and Engineering Physics, Cornell University, Ithaca, NY, USA \\ 2. Department of Chemistry, University of Chicago, Chicago, IL, USA \\ 3. School of Materials Science and Engineering, Ulsan National Institute of Science and \\ Technology, Ulsan, Republic of Korea \\ 4. James Franck Institute, University of Chicago, Chicago, IL, USA \\ 5. Institute for Molecular Engineering, University of Chicago, Chicago, IL, USA \\ 6. Kavli Institute for Nanoscale Science, Cornell University, Ithaca, NY, USA \\ * Corresponding author: david.a.muller@cornell.edu
}

Dark field TEM (DFTEM) is a simple yet effective technique for imaging crystal grain structure of polycrystalline materials. Collecting the signal from different diffracted spots can create a colored patchwork of grains in a material [1]. This is straightforward when crystal lattices differ by just an in-plane rotation, such as is the case for monolayers of flat 2D materials. However, we construct a new 3D structure by directly growing monolayer $\mathrm{MoS}_{2}$ onto a corrugated substrate (Fig. 1a). While flat monolayer $\mathrm{MoS}_{2}$ can be grown as a single crystal, the topology of these corrugations cannot be accommodated with a uniform crystal structure (Fig. 1c).

Furthermore, the 3D structure of the corrugations means that various parts of the sample are at different tilts. Tilt causes the diffraction patterns (Fig 2d) to distort asymmetrically compared to a pattern found in normal flat $\mathrm{MoS}_{2}$ (Fig 2e). This asymmetrical distortion caused by tilt changes both the measured distance and rotation of the diffracted spots, obfuscating proper measurement of strain and in-plane rotation. This means traditional dark field imaging methods are less effective as the diffracted spots move in various ways when the crystal is tilted, rotated, and strained. The effect of the tilt on the diffraction pattern must be separated for proper analysis.

Rather than measuring the signal of a selected diffracted spot, we operate in STEM mode and collect the full diffraction pattern, including the central beam, at every scanning point using the Electron Microscope Pixel Array Detector (EMPAD) [2]. We use a small convergence angle for a more parallel beam, so that the diffracted spots do not overlap, and we can properly sample the local crystal structure at each scanning point. We track the six primary diffracted spots of the diffraction pattern and map them to a transformation of a flat unstrained $\mathrm{MoS}_{2}$ crystal using three Euler angles and a biaxial strain. This allows us to successfully decouple in-plane rotation from the out-of-plane tilt and map strain of such nonconventional 2D crystals. Using this mapping technique at various corrugation sizes (Fig. 2a,b,c) shows that there is a critical size around 160 $\mathrm{nm}$ where the corrugations change from a curved structure with a continuous shift in crystal rotation to a more facetted shape, creating a larger plateau in the center. This shift from spherical to faceted morphology can be explained analytically as a balance between the energy cost of introducing lines of structural defects and the strain inherent to the curved structure $[3,4]$.

References: 
[1] P.Y. Huang et al., Nature 469 (2011) p. 389-392

[2] M. W. Tate et al., Microscopy and Microanalysis 22 (2016), p. 237-249.

[3] D.J. Srolovitz et al., Physical Review Letters 74 (1995), p. 1779

[4] Funding from the DOD/AFOSR 2D MURI project (FA9550-16-1-0031) and facilities support from the Cornell Center for Materials Research, an NSF MRSEC (DMR-1719875).

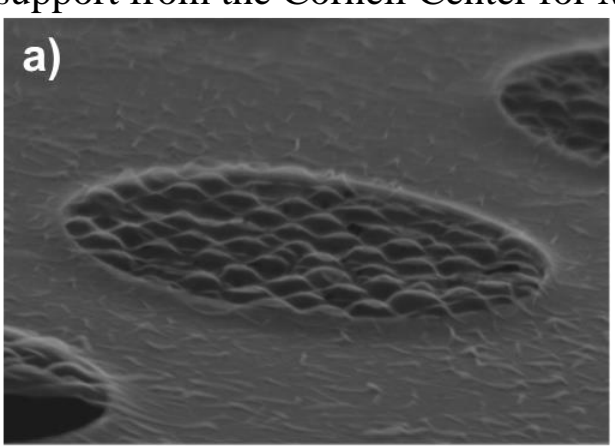

$500 \mathrm{~nm}$

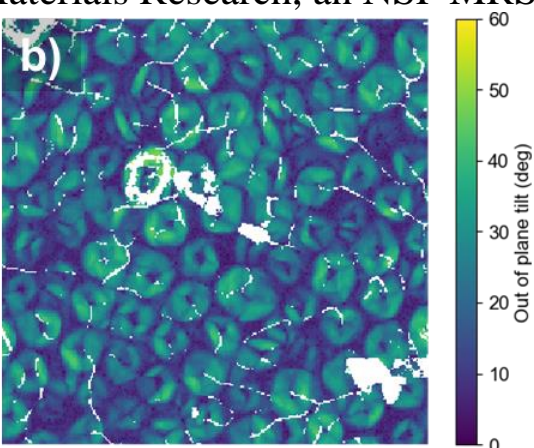

$200 \mathrm{~nm}$

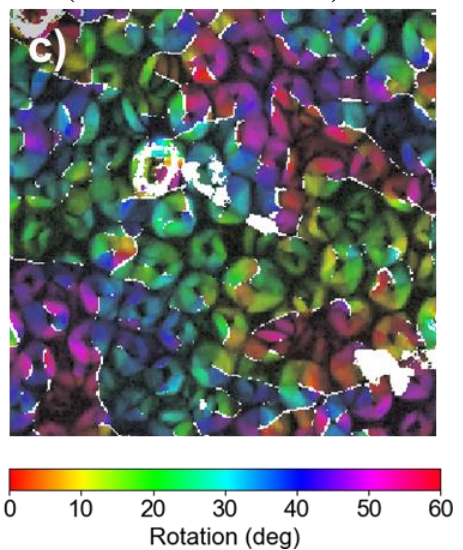

Figure 1. a) SEM image of corrugated $\mathrm{MoS}_{2}$. The corrugations are approximately $50 \mathrm{~nm}$ in diameter and $25 \mathrm{~nm}$ in height. b) EMPAD quantitative tilt map of the $50 \mathrm{~nm}$ corrugations shows the local out-of-plane tilt of the crystal. c) Tilt + rotation maps with the color from the in-plane rotation of the crystal and the saturation from the tilt shown in b). White pixels represent areas which could not be mapped. These maps demonstrate that the corrugated, curved structure cannot be tiled by a single crystal without introducing topologically necessary defects.
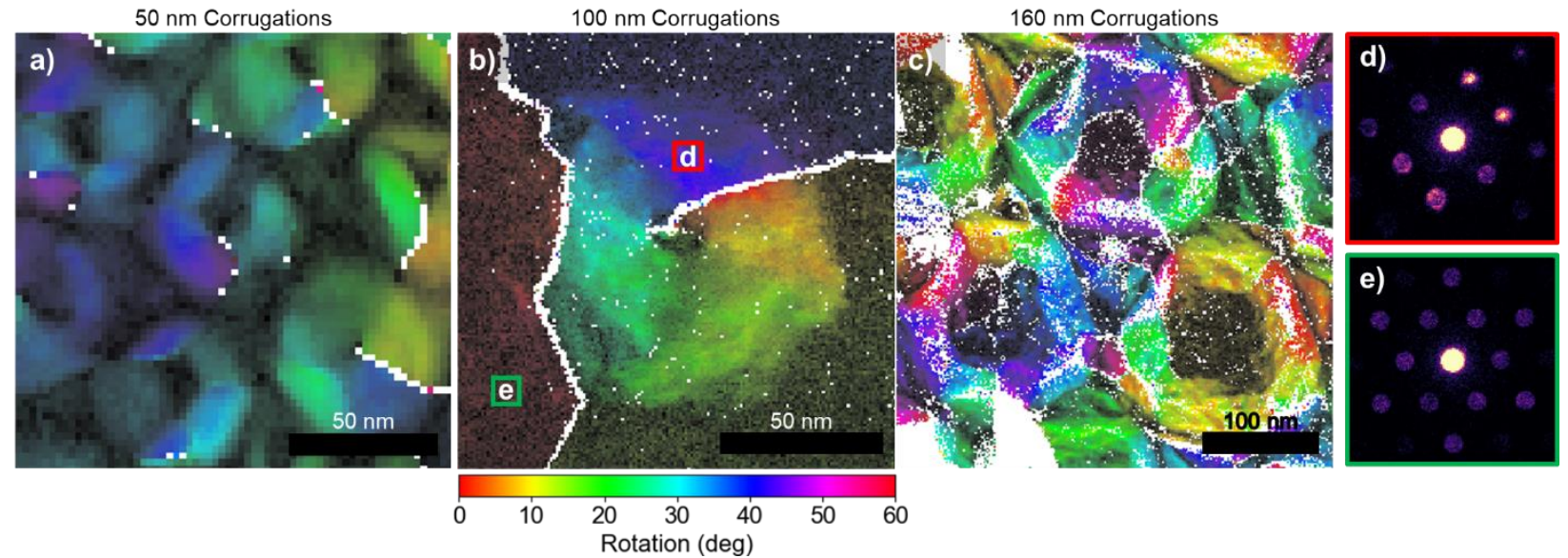

Figure 2. a,b,c) Tilt + rotation maps of corrugations at various sizes of $50 \mathrm{~nm}, 100 \mathrm{~nm}$, and 160 $\mathrm{nm}$ diameters respectively. While all corrugations require different crystal orientations to accommodate the curved topology, around the critical size of $160 \mathrm{~nm}$, the corrugation takes a more facetted shape and there is a larger plateau in the center. $d, e)$ Diffraction patterns taken from $2 b$ ) at spots indicated by the colored square borders. The pattern at d) is asymmetrically distorted due to the tilt on the side of the corrugation while the pattern at e) is the typical pattern found in flat $\mathrm{MoS}_{2}$. Figures were generated from diffraction data collected on the EMPAD at $80 \mathrm{keV}$. The 50 $\mathrm{nm}$ sample was done with a $1.35 \mathrm{mrad}$ aperture; the $100 \mathrm{~nm}$ and $160 \mathrm{~nm}$ samples were done with a $2.96 \mathrm{mrad}$ aperture. 